\title{
The Red Giant Branch in the Tycho-2 Catalogue
}

\author{
G. A. Gontcharov*
}

July 9,2021

Pulkovo Astronomical Observatory, Russian Academy of Sciences, Pulkovskoe sh. 65, St. Petersburg, 196140 Russia

Key words: color-magnitude diagram, Galactic solar neighborhoods, giant and subgiant stars, proper motions and radial velocities.

Based on multicolor photometry from the 2MASS and Tycho-2 catalogues, we have produced a sample of 38368 branch red giants that has less than $1 \%$ of admixtures and is complete within $500 \mathrm{pc}$ of the Sun. The sample includes $30671 \mathrm{~K}$ giants, $7544 \mathrm{M}$ giants, $49 \mathrm{C}$ giants, and 104 suspected supergiants or S stars. The photometric distances have been calculated for $\mathrm{K}$, $\mathrm{M}$, and C stars with an accuracy of $40 \%$. Tycho-2 proper motions and PCRV radial velocities are used to analyze the stellar kinematics. The decrease in the stellar distribution density with distance from the Galactic equator approximated by the barometric law, contrary to the Besancon model of the Galaxy, and the kinematic parameters calculated using the OgorodnikovMilne model characterize the overwhelming majority of the selected $\mathrm{K}$ and M giants as disk stars with ages of more than 3 Gyr. A small number of K and $\mathrm{M}$ giants are extremely young or, conversely, thick-disk ones. The latter show a nonuniform distribution in the phase space of coordinates and velocities, arguing against isothermality and full relaxation of the disk and for the theory of dynamical streams or superclusters. The spatial distribution and kinematics of the selected $\mathrm{C}$ stars force us to consider them as asymptotic branch giants with masses of more than $2 M_{\odot}$ and ages of less than 2 Gyr probably associated with the Gould Belt. The offset of the Sun above the Galactic equator has been found from the distribution of stars to be $13 \pm 2$

*E-mail: georgegontcharov@yahoo.com 
pc, which coincides with the previously obtained value for the clump red giants. 


\section{INTRODUCTION}

In this paper, we continue to investigate the spatial distribution and kinematics of stars of various types within the kiloparsec of the Galaxy nearest to the Sun. Previously, based on multiband photometry from the Tycho-2 (Høg et al. 2000) and 2MASS (Skrutskie et al. 2006) catalogues, Tycho-2 proper motions, and PCRV radial velocities (Gontcharov 2006) and using calibrations based on data from the Hipparcos catalogue, the first (ESA 1997) and new (van Leeuwen 2007) versions, we produced and analyzed samples of OB stars (Gontcharov 2008a), red giant clump (RGC) stars (Gontcharov 2008b), and subdwarfs (Gontcharov 2010). These works, along with the corresponding Monte Carlo simulations (Gontcharov 2009a), showed that stars of some types could be successfully selected from modern photometric and astrometric surveys without any spectral classification for their further studies.

Here, we selected and investigated stars belonging to the red giant branch (RGB) in its reddest part, i.e., giants with effective temperatures lower than those for RGC ones. According to the spectral classification, the selected stars are of types later than K3I-K3III. However, the spectral classification is known only for some of the selected stars and was not used here to determine any quantities.

\section{THE SELECTION OF STARS}

\section{Input Photometry}

The accuracy of the photometry in Tycho-2 (the $B_{T}$ and $V_{T}$ bands) and 2MASS (the infrared (IR) $J, H$, and $K s$ bands) deteriorates for faint stars. For the selection of RGB stars, we used 718000 stars with $B_{T}<11.7^{m}$ and $V_{T}<11^{m}$. The median accuracy of their photometry is $\sigma\left(B_{T}\right)=0.04^{m}$, $\sigma\left(V_{T}\right)=0.03^{m}$. We imposed no constraints on the $J, H$, and $K s$ magnitudes, because here we consider red stars that are rather bright in the infrared. At $K s>5.5^{m}$, the median accuracy is $\sigma(J)=0.02^{m}, \sigma(H)=0.03^{m}, \sigma(K s)=$ $0.02^{m}$.

However, the near-IR photometry for the brightest stars $\left(K s<5.5^{m}\right)$ is inaccurate, but there is accurate far-IR photometry for them from the IRAS catalog (IRAS 1988). We cross-identified Tycho-2 and IRAS using the SIMBAD database in Strasbourg. For red giants with accurate photometry, 
the $K s$ magnitude was calibrated as a function of the logarithm with base 2.512 of the IR flux at wavelength 12 microns (below referred to as $F 12$ ) taken from IRAS: $\left.\left.K s=0.0087\left(\log _{2.512}(F 12)\right)^{3}\right)-0.0371\left(\log _{2.512}(F 12)\right)^{2}\right)-$ $\left.0.95\left(\log _{2.512}(F 12)\right)\right)+4.2$. The calibration gave $\sigma(K s)=0.18^{m}$. Owing to this calibration, all bright stars were retained in the sample.

In the sample, we retained only single stars and bright components of

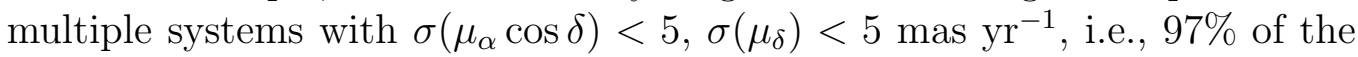
sky stars brighter than $B_{T}=11.7^{m}$ and $V_{T}=11^{m}$. The median accuracy is

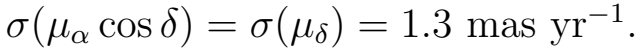

\section{The $\left(V_{T}-K s\right)-\left(B_{T}-V_{T}\right)$ Diagram}

We selected and classified RGB stars using two-color diagrams, primarily the $\left(V_{T}-K s\right)-\left(B_{T}-V_{T}\right)$ diagrams with the most accurate color indices. Other two-color diagrams and diagrams with three-color indices $\mathrm{Q}$ were used for checking.

Part of the $\left(V_{T}-K s\right)-\left(B_{T}-V_{T}\right)$ diagram with the RGC and the RGB is shown in Fig. 1. To present the theoretical data on this diagram, we recalculated the Johnson $B_{j}, V_{j}$, and $K_{j}$ magnitudes to the $B_{T}, V_{T}$, and $K s$ magnitudes by taking into account the relations $\left(B_{T}-V_{T}\right)=\left(B_{j}-V_{j}\right) / 0.78$ for giant stars (Gontcharov 2008b) and $\left(V_{T}-K s\right)=\left(V_{j}-K_{j}\right)+0.09\left(B_{T}-V_{T}\right)$ (ESA 1997). Both relations introduce an error of no more than $0.05^{\mathrm{m}}$.

The main sequence and the giant branch on this diagram are not separated. However, as we showed previously (Gontcharov 2009a), there are actually no dwarfs among the reddest Tycho- 2 stars, because Tycho- 2 is limited in apparent magnitude (below, we discuss the elimination of the remaining dwarfs as well). To produce a pure sample of RGB stars free not only from dwarfs but also from RGC stars, we imposed the constraints $(J-K s)>0.8-\sigma(J-K s),\left(B_{T}-V_{T}\right)>1.2^{m},\left(V_{T}-K s\right)>2.8$ and $\left(B_{T}-V_{T}\right)>1.86-0.086\left(V_{T}-K s\right)$ on the sample (the last two conditions are indicated by the dashed straight lines in the left bottom part of the figure) that follow from the theoretical evolutionary tracks by Girardi et al. (2000) for stars older than 1 Gyr (the younger RGC stars of the color under consideration are extremely rare).

These constraints do not eliminate noticeably reddened RGC stars. The following approach was applied for them. The reddening line of length $E_{\left(B_{T}-V_{T}\right)}=0.5^{m}$ is indicated by the vector in the left part of the figure according to Gontcharov (2008a). The "cloud" of points in the figure is 
smeared by the reddening in this direction. Assuming the reddening of stars at high latitudes to be small $\left(E_{\left(B_{T}-V_{T}\right)}<0.3^{m}\right)$ and selecting such stars, we can calculate the average dependence of $\left(B_{T}-V_{T}\right)$ on $\left(V_{T}-K s\right)$ for them. It is not shown in the figure, because it coincides with the theoretical and mean empirical colors obtained by Pickles (1998) based on the spectral energy distribution for unreddened solar-metallicity K3III, K4III, K5III, M0III, M1III, M2III, M3III, M4III, M5III, M6III, M7III, M8III, M9III, M10III stars that are indicated by the large open squares in the figure, respectively, from left to right. Shifting this dependence along the reddening line, we obtain the line of normal colors for $\mathrm{M}$ stars indicated in the figure by the thin solid black curve below the main cloud of points: $\left(B_{T}-V_{T}\right)=0.0183\left(V_{T}-K s\right)^{3}-0.3594\left(V_{T}-K s\right)^{2}+2.15\left(V_{T}-K s\right)-2.3$. Taking into account the accuracy of the photometry, we obtain the line below which the stars cannot belong to the RGB and were rejected as reddened RGC stars: $\left(B_{T}-V_{T}\right)=0.0183\left(V_{T}-K s\right)^{3}-0.3484\left(V_{T}-K s\right)^{2}+2\left(V_{T}-K s\right)-2$. This polynomial is indicated by the dashed line at the bottom of the figure. The selected RGB stars are indicated in the figure by the black symbols and the rejected extraneous stars, mostly the RGC ones, are indicated by the gray points below and leftward of the three dashed lines.

An important feature of this diagram is the kink in the dependence of $\left(B_{T}-V_{T}\right)$ on $\left(V_{T}-K s\right)$ for RGB stars seen in the figure that separates the $\mathrm{K}$ and $\mathrm{M}$ stars. This nonlinearity of the dependence is explained by a difference in spectral energy distribution for $\mathrm{K}$ and $\mathrm{M}$ stars, namely the presence of strong absorption lines in the spectra of $\mathrm{M}$ stars in the $V_{T}$ band.

According to Pickles (1998), the nonsolar metallicity stars and bright giants at the level of accuracy under consideration are lost among the solar metallicity giants and cannot be distinguished on this diagram. In contrast, according to the Tycho Spectral Types (TST) catalogue (Wright et al. 2003), the supergiants and S stars (inseparable photometrically and below designated together as $\mathrm{S} /$ supergiants) and carbon stars (below referred to as $\mathrm{C}$ ones) must have slightly larger $\left(B_{T}-V_{T}\right)$ color indices at the same $\left(V_{T}-K s\right)$. Although an admixture of highly reddened or peculiar KIII and MIII stars is possible here, we, nevertheless, distinguished two groups of stars: (a) more likely $\mathrm{C}$ stars (marked by the diamonds at the top of the figure) and (b) S/supergiants (marked by the filled squares). Taking into account the spectral energy distribution, we assume that a star is more likely a $\mathrm{C}$ one than an $\mathrm{S} /$ supergiant one when all of the following conditions are met: $\left(B_{T}-V_{T}\right)>0.27\left(V_{T}-K s\right)+1.14$ (indicated by the 
thick gray straight line at the top of the figure), $(J-K s)>1.35^{m}$ and $\left(V_{T}-J\right)<7(J-H)-1.9$. The suspected $\mathrm{S} /$ supergiants and $\mathrm{C}$ stars are separated from the KIII and MIII stars by the polynomial $\left(B_{T}-V_{T}\right)=$ $0.009\left(V_{T}-K s\right)^{3}-0.1828\left(V_{T}-K s\right)^{2}+1.1552\left(V_{T}-K s\right)+0.2$. The latter is indicated in the figure by the thick gray curve and actually reflects a reasonable assumption about the interstellar extinction in the region of space under consideration: $A_{V}<3^{m}$. The KIII and MIII stars were separated along the reddening line $\left(B_{T}-V_{T}\right)=0.365\left(V_{T}-K s\right)+0.25$ (the gray straight line at the center of the figure), although this separation is arbitrary, because the properties of stars change gradually in the sequence of $\mathrm{K}-\mathrm{M}$ types.

\section{The Elimination of Dwarfs}

To eliminate dwarfs, we used reduced proper motions: $M_{V T}^{\prime}=V_{T}+5+5 \cdot \lg (\mu)$ and the analogous $M_{K s}^{\prime}$, where $\mu=\left(\mu_{\alpha}^{2} \cos \delta+\mu_{\delta}^{2}\right)^{1 / 2}$ is the proper motion in arcseconds. The constraints $M_{V T}^{\prime}<8.7$ and $M_{K s}^{\prime}<5$ were imposed on the sample. In this way, we eliminated 44 suspected dwarfs. As expected, the number of dwarfs in the range of colors under consideration is very small. Thus, RGB stars were actually selected using only the colors, as distinct from the selection of RGC stars in Gontcharov (2008b), where the reduced proper motions played a key role.

\section{CHARACTERISTICS OF STARS}

\section{Reddening and Extinction}

The reddening $E_{\left(B_{T}-V_{T}\right)}$ and extinction $A_{V}$ (the extinctions in the $V_{j}$ and $V_{T}$ bands are equal with a sufficient accuracy) for the stars under consideration were used to calibrate the absolute magnitudes $M_{V T}$ from the $\left(V_{T}-K s\right)$ color and to calculate the photometric distances $R_{p h}$ from the formula

$$
\log \left(R_{p h}\right)=\left(V_{T}-M_{V T}+5-A_{V}\right) / 5 .
$$

As we show below, in both cases, the accuracy is determined by the natural scatter $\sigma\left(M_{V T}\right)=0.8^{m}$, not by a much more accurate correction for the extinction.

The extinction for each selected star was estimated by three different methods: 
- From the formula $A_{E B V}=3.1 E_{\left(B_{T}-V_{T}\right)}$, where the reddening $E_{\left(B_{T}-V_{T}\right)}$ was calculated as the shift of the star from the line of normal colors mentioned above. Given the accuracy of the photometry and variations in the extinction coefficient of about 3.1, the accuracy of this extinction estimate is $\sigma\left(A_{E B V}\right) \approx 0.3^{m}$.

- From the deviation of the spectral energy distribution for the star from the unreddened one, i.e., from a linear combination of photometric magnitudes. Previous (Gontcharov 2008b), we showed that the extinction $A_{B V J K}=0.96\left(-1.89 B_{T}+2.89 V_{T}+0.25 J-1.25 K s-0.26\right)$. For stars without strong lines in their spectra, the accuracy of this estimate is determined by the accuracy of the photometry and is also $\sigma\left(A_{B V J K}\right) \approx 0.3^{m}$.

- As a function of the Galactic coordinates, $A_{G}=f\left(l, b, R_{p h}\right)$, from our three-dimensional extinction model (Gontcharov 2009b). In this case, $A_{G}$ and $R_{p h}$ are refined by iterations: $M_{V T}$ is calibrated from $\left(V_{T}-\right.$ $K s)$, as is shown below; subsequently, we calculate $\log \left(R_{p h}\right)=\left(V_{T}-\right.$ $\left.M_{V T}+5\right) / 5$ in the first approximation, then calculate $A_{G}=f\left(l, b, R_{p h}\right)$, and recalculate $R_{p h}$ in the second approximation using Eq. (1). The iterations converge rapidly, because $A_{V}$ in Eq. (1) is small compared to the remaining quantities. Within $500 \mathrm{pc}$ of the Sun (i.e., for most of the stars under consideration), the accuracy of the model $\sigma\left(A_{G}\right) \approx$ $0.3^{m}$ was estimated previously (Gontcharov 2009b) by comparing it with three catalogs of individual extinctions for stars. We also showed in the same paper that the accuracy of correction for the extinction $0.3^{m}$ typical of all methods is a difficult-to-surmount limit due to the corresponding natural scatter of individual extinctions for neighboring stars.

Comparison of $A_{E B V}, A_{B V J K}$ and $A_{G}$ for the selected stars showed the following:

- $A_{E B V}$ is implausibly large for $\mathrm{C}$ stars and $\mathrm{S} /$ supergiants, because the relation between $\left(B_{T}-V_{T}\right)$ and $\left(V_{T}-K s\right)$ is different from that for $\mathrm{K}$ and $\mathrm{M}$ stars;

- because of strong spectral lines, $A_{B V J K}$ is implausibly large for many of the M stars and $\mathrm{S} /$ supergiants and is negative for many of the $\mathrm{C}$ stars; 
- $A_{G}$ has been overestimated for stars farther than 500 pc, outside the Gould Belt: for example, the mean differences for $\mathrm{K}$ stars $\overline{A_{G}-A_{B V J K}}=$ $\overline{A_{G}-A_{E B V}}=0.3^{m}$;

- for the remaining stars, different extinction estimates agree at the level of the mentioned limit $0.3^{m}$ : the standard deviation is $\sigma\left(A_{G}-\right.$ $\left.A_{B V J K}\right)=0.3^{m}$ for $\mathrm{K}, \mathrm{C}$ stars and $\mathrm{S} /$ supergiants within $500 \mathrm{pc}$ of the Sun, $\sigma\left(A_{G}-A_{E B V}\right)=0.3^{m}$ for $\mathrm{K}$ and $\mathrm{M}$ stars within the same radius, and $\sigma\left(A_{B V J K}-A_{E B V}\right)=0.3^{m}$ for $\mathrm{K}$ stars in the entire region of space.

Below, we use $A_{E B V}$ for $\mathrm{K}$ and $\mathrm{M}$ stars and $A_{G}$ for $\mathrm{C}$ stars. No extinction was used for $\mathrm{S} /$ supergiants, because $M_{V T}$ and $R_{p h}$ were not calculated due to the scarcity of data for the calibration of $M_{V T}$ from $\left(V_{T}-K s\right)$.

\section{Absolute Magnitudes and Distances}

There are 38368 RGB stars in the final sample. The new Hipparcos reduction (van Leeuwen 2007) contains 12208 of the selected stars, but only 2900 of them have parallaxes with a relative accuracy better than 20\%: $536 \mathrm{M}$ stars, $2354 \mathrm{~K}$ stars, $9 \mathrm{C}$ stars, and $1 \mathrm{~S}$ star. We used them to calculate the absolute magnitude $M_{V T}$, which was then calibrated from the $\left(V_{T}-K s\right)$ color. In this case, $M_{V T}$ and $\left(V_{T}-K s\right)$ were corrected for the previously found extinction and reddening with $E_{\left(V_{T}-K s\right)}=2.74 E_{\left(B_{T}-V_{T}\right)}$ from Fitzpatrick and Massa (2007), who also estimated the relative accuracy of this relation to be better than $2 \%$.

To estimate the effects of the Malmquist and Lutz-Kelker biases (Perryman 2009, pp. 209-211) on the dependence of $M_{V T}$ on distance and $\left(V_{T}-K s\right)$, we performed Monte Carlo simulations. We used Gaussian and uniform distributions implemented by the Microsoft Excel 2007 random number generator, whose general description was given by Wichman and Hill (1982). We generated 65000 model stars for which we specified the following:

- a uniform distribution in rectangular Galactic coordinates $X$ and $Y$ within $1100 \mathrm{pc}$ of the Sun and a Gaussian distribution in $Z$ with a standard deviation of $200 \mathrm{pc}$ (this corresponds with a sufficient accuracy to the distribution of comparatively old disk stars according to the Besancon model of the Galaxy (BMG) (Robin et al. 2003));

- a uniform distribution in the range $3^{m}<\left(V_{T}-K s\right)<8^{m}$. 
Subsequently, we calculated the following:

- $M_{V T}$ with a mean $-0.11\left(V_{T}-K s\right)^{3}+2\left(V_{T}-K s\right)^{2}-11.4\left(V_{T}-K s\right)+20.4$ and a standard deviation of $0.8^{m}$ in accordance with the theoretical isochrones for a mixture of stars with ages of $10^{9}-10^{10} \mathrm{yr}$ (Girardi et al. 2000) with allowance made for $V_{T} \approx V_{j}+0.2^{m}$;

- the true distance $R=\left(X^{2}+Y^{2}+Z^{2}\right)^{1 / 2}$, the Galactic coordinates $\tan (l)=Y / X, \tan (b)=Z /\left(X^{2}+Y^{2}\right)^{1 / 2}$,

- the interstellar extinction $A_{V}$ from our analytical extinction model (Gontcharov 2009b) and the reddening $E_{\left(V_{T}-K s\right)}=A_{V} / 3.1 \cdot 2.74$;

- $V_{T}=M_{V T}-5+5 \lg (R)+A_{V}$ and the photometric error $\sigma\left(V_{T}\right)=$ $0.01 e^{0.3 V_{T}}$ in accordance with the Tycho- 2 characteristics;

- the true parallax $\pi=1000 / R$ (where $\mathrm{R}$ in pc and $\pi$ in mas) and its measured value with a standard deviation of 1 mas relative to the true value.

In accordance with the limitation of the Hipparcos catalogue (ESA 1997, p. 131), we retain only the stars with $V_{T}<7.3+1.1|\sin (b)|$ and only the stars with a relative accuracy of the parallax better than $20 \%$.

Our simulations showed both a considerable decrease in the mean value of $M_{V T}$ and some change in the dependence of $M_{V T}$ on $\left(V_{T}-K s\right)$ with distance. However, for stars with $\pi>3.3$ mas these effects do not exceed $0.1^{m}$. This conclusion was confirmed by our analysis of the change in the dependence of $M_{V T}$ on $\left(V_{T}-K s\right)$ with distance for the real selected $\mathrm{K}$ and $\mathrm{M}$ stars.

As a result, we calibrated $M_{V T}$ as a function of $\left(V_{T}-K s\right)$ for $2167 \mathrm{~K}$ and M stars with a relative accuracy of the parallax better than $20 \%$ and $\pi>3.3$ mas: the calibration curve $M_{V T}=0.124\left(V_{T}-K s\right)^{2}-1.16\left(V_{T}-K s\right)+2.24$ found is shown in Fig. 2 as the thick black line. For $\mathrm{C}$ stars, we adopted the same dependence but brighter by $1^{m}$. In Fig. 2, the $\mathrm{K}$ and $\mathrm{M}$ stars used for the calibration are marked by the crosses and snowflakes, respectively. The $\mathrm{C}$ stars (circles) and one $\mathrm{S}$ star (square) with a relative accuracy of the parallax better than $20 \%$ that were not used for the calibration are also marked here.

For comparison with the observational data, Fig. 2 shows the theoretical isochrones for solar-metallicity stars from Girardi et al. (2000): the dashed and solid thin curves indicate the RGB and the asymptotic giant branch, respectively; the upper dashed and solid curves correspond to an age of $10^{9}$ 
yr and the lower curves correspond to $10^{10} \mathrm{yr}$. Stellar masses of about 2.3 $M_{\odot}$ for $10^{9} \mathrm{yr}$ and about $1.1 M_{\odot}$ for $10^{10} \mathrm{yr}$ correspond to the segments of the isochrones falling into the region filled with the selected stars shown in the figure. The massive young stars of the sample are apparently on the asymptotic giant branch, while the low-mass old ones are on the RGB. The theoretical isochrones do not quite correspond to the positions of the reddest stars from the sample, which is apparently indicative of shortcomings of the theory. On the whole, the positions of the selected stars relative to the isochrones suggest that disk stars with ages from 1 to 10 Gyr dominate in the sample. The picture is complicated by the fact that metallicity variations displace significantly the stars and isochrones. As a result, it is difficult to draw conclusions about the age and metallicity of a specific star from its position on the diagram.

For K, M, and C stars, the standard deviation of $M_{V T}$ from the calibration curve is $0.8^{m}$. This allows us to calculate the photometric distances $R_{p h}$ from Eq. (1) with a relative accuracy of $40 \%$ and the corresponding rectangular Galactic coordinates $X, Y, Z$.

\section{Comparison with Known Classification}

Out of the 38368 selected stars, 31049 (81\%) have spectral classification either from the Hipparcos Input Catalogue (HIC) (Turon et al. 1993) or from the TST. For many stars, the classification from these catalogues differs or is designated as dubious. Among the stars selected as S/supergiants, five were previously classified as type $\mathrm{C}$. This classification can apparently be trusted and below we consider them to be $\mathrm{C}$ stars. As a result, we attributed 30671 , 7544, 104, and 49 stars to types K, M, S/supergiants, and C, respectively.

The main statistics of the spectral classification is the following: 6029 of the 7544 stars selected as type M have the old classification, 5200 (86\%) of them were classified as $\mathrm{M}$ at least in one catalogue, and most of the remaining stars were classified as K; 24872 of the 30671 stars selected as type $\mathrm{K}$ have the old classification, $21589(87 \%)$ of them were classified as K at least in one catalogue, and most of the remaining stars were classified as $\mathrm{M}$; for S/supergiant, these numbers are, respectively, 104, 99, and 45 (45\%) (for many of the remaining stars, the luminosity class is unknown); for type C, the numbers are 49, 49, 46 (94\%). The correctness of the classification for

the selected S/supergiants is confirmed by their strong concentration to the Galactic plane: $|b|<20^{\circ}$ for all stars. Thus, since the derived classifica- 
tion for the overwhelming majority of the selected stars coincides with the old one, the applied method of classification based on broadband multicolor photometry should be recognized as successful.

Moreover, for some stars, the new classification based on photometry is more accurate than the old spectral one. For example, 191 and 135 selected stars were previously classified as dwarfs and subgiants, respectively. This classification cannot be trusted, because the Hipparcos parallaxes for 56 dwarfs and 30 subgiants are known with a relative accuracy better than $50 \%$. This allows their luminosity class to be checked by the absolute magnitude: on average, $\overline{M_{V T}}=-0.1 \pm 1.2$ for dwarfs and $\overline{M_{V T}}=0.4 \pm 1.2$ for subgiants. These values correspond to those for giants and are totally unsuitable for dwarfs $\left(\overline{M_{V T}} \approx 8\right)$ and subgiants $\left(\overline{M_{V T}} \approx 3\right)$ according to Hipparcos data.

\section{The Spatial Distribution of Stars}

The distribution of the selected stars in projection onto the $X Y, X Z$, and $Y Z$ planes is shown in Fig. 3: (a) type $\mathrm{K}$, (b) type M, and (c) type C. The radius of the region where the sample is complete can be estimated from the change in the stellar distribution density and from the maximum of the mean interstellar extinction that is reached at the edge of this region: further out, the mean extinction decreases, because the sample becomes incomplete mainly through the loss of stars with large extinction. Both approaches give the same estimate: for all types of stars, the sample is complete up to $500 \mathrm{pc}$. In this space, the distribution density of $\mathrm{K}$ and $\mathrm{M}$ stars decreases toward the Galactic anticenter as for typical disk stars in accordance with the BMG. In contrast, $\mathrm{C}$ stars dominate in the second and third Galactic quadrants and are encountered here at greater distances than in the first and fourth quadrants. The Gould Belt stars are distributed precisely in this way (Perryman 2009, pp. 324-328). For this reason, below we discuss the evolutionary status and ages of $\mathrm{C}$ stars.

"Flaws" are noticeable in the distribution of stars of all types on the $X Z$ plots because of the interstellar extinction in the Gould Belt, which is oriented roughly in this plane with an inclination of $17^{\circ}$ to the equator (Gontcharov 2009b).

The decrease in the stellar distribution density with distance from the equator can theoretically be proportional to $\operatorname{sech}^{2}\left(2 Z / Z_{0}\right)$ (Girardi et al. $2005)$ or correspond to the complex dependence from the BMG or the barometric law $D_{0} \cdot e^{-|Z| / Z_{0}}$, where $D_{0}$ is the density in the equatorial plane 
and $Z_{0}$ is the distance from this plane at which the density decreases by a factor of $e$ or the half-thickness of a homogeneous layer of stars (Parenago 1954, p. 264). Figure 4 shows the distribution of the selected (a) K and (b) $\mathrm{M}$ stars as a function of the $Z$ coordinate in the vertical cylinder elongated along the $Z$ axis with a radius of 400 pc around the Sun and the curves of the bolometric law fitting best this distribution: $D_{0}=1262, Z_{0}=263$ pc for type $\mathrm{K}$ and $D_{0}=555, Z_{0}=217 \mathrm{pc}$ for type $\mathrm{M}$. The values of $Z_{0}$ found are typical of rather old Galactic disk stars according to the BMG. The lower value for $\mathrm{M}$ stars most likely suggests not their relative youth but the possible contamination of their sample by young forming stars, C stars, and supergiants. This fit closely corresponds to the observed distribution at all $Z$, except for the region $-100<Z<100$ pc, where less than 100 stars of each type produce an excess that can also be fitted by the barometric law with $Z_{0}=50 \mathrm{pc}$. These stars are apparently an admixture of young stars. This excess near the equator cannot be removed even by reducing the $Z$ range used for fitting. It is also important that fitting the data by the square of the hyperbolic secant or by the dependence from the BMG for any reasonable characteristics of the thin and thick disks does not give a better result than the barometric law. Thus, because of their comparatively small number, the thick-disk giants do not manifest themselves clearly among the selected stars. However, having extreme velocities, as we show below, they manifest themselves when averaging the kinematic characteristics of the stars in the same spatial cells.

The discrepancy between the $Z$ distribution found and the dependence from the BMG apparently suggests the absence of isothermality and full relaxation of the Galactic disk assumed in the BMG. This is an expected result in connection with the detection of several kinematically isolated groups among comparatively old red giants that are superclusters or dynamical streams by Famaey et al. (2005).

The asymmetric distribution of stars in the cylinder under consideration southward and northward of the Galactic equator allows the offset of the Sun to be calculated: $13 \pm 2 \mathrm{pc}$; this corresponds to the value found previously (Gontcharov 2008b) from RGC stars.

\section{Stellar Kinematics}

The present views of the Galactic evolution suggest the relationship between the age of stars, their metallicity, the distance from the Galactic equator, the 
velocity dispersion, and the velocity component $V$.

The proper motions and radial velocities allow one to calculate the velocities of stars in Galactic $U, V, W$ coordinates and to analyze their kinematics by comparing the results with the analysis of a sample of red giants from Hipparcos (Famaey 2005) and a sample of RGC stars from Tycho-2 (Bobylev et al. 2009).

The radial velocities $V_{r}$ from the PCRV catalog are known with an accuracy better than $5 \mathrm{~km} \mathrm{~s}^{-1}$ for the 3653 selected stars: $2737 \mathrm{~K}, 852 \mathrm{M}, 41$ $\mathrm{C}$, and $23 \mathrm{~S} /$ supergiant stars (since the distances for the $\mathrm{S} /$ supergiants were not calculated, they are not considered below). The absence of observational selection in the distribution of these stars on the celestial sphere frees the kinematic characteristics being determined from biases, as distinct from the sample by Famaey et al. (2005) containing only Northern-Hemisphere stars.

The accuracy of the calculated $U, V, W$ is actually determined by the accuracy of the derived distances. It is about $15 \mathrm{~km} \mathrm{~s}^{-1}$ for each velocity component, when using both trigonometric and photometric distances. This also determines the accuracy of the kinematic parameters obtained below.

Consider the dependences of the mean velocity components and velocity dispersions on $Z$ coordinate in the vertical cylinder elongated along the $Z$ axis with a radius of $500 \mathrm{pc}$ around the Sun for (a) type K and (b) type $\mathrm{M}$ shown in Fig. 5. The accuracy is indicated by the vertical bars. The results from photometric distances and Hipparcos parallaxes with a relative accuracy of the parallax better than $20 \%$ are indicated by the solid and dashed lines, respectively. We see that everywhere, except the extreme $Z$, the results from the two types of distances agree well between themselves. The disagreements at the extreme $Z$ are explained by the influence of few thick-disk (and, possibly, halo) stars with extreme velocities located at the boundary of the region of space under consideration and included or not included in the sample depending on whether the trigonometric or photometric distances are used.

The same stars causes a nonuniform decrease in the velocity component $V$ and an increase in the velocity dispersion with distance from the equator, for example, at $-450<Z<-350 \mathrm{pc}$ for $\mathrm{K}$ stars and $Z \approx-450,-250$, and $-50 \mathrm{pc}$ for $\mathrm{M}$ stars. The few (among the selected stars) thick-disk (and, possibly, halo) giants, which, as has been mentioned above, are distributed in the phase space of coordinates and velocities as isolated groups, dynamical streams or superclusters (Famaey et al. 2005), manifest themselves in this way. However, disk giants younger than 10 Gyr dominate in the entire region 
of space under consideration: the velocities and velocity dispersions averaged over the spatial cells are almost everywhere within the limits established by the BMG for thin-disk stars $(\sigma(U)<50, \sigma(V)<35, \sigma(W)<25, V>-35$ $\left.\mathrm{km} \mathrm{s}^{-1}\right)$. In subsequent studies, we will consider specific stars and groups of stars from the thick disk that cause oscillations of the mean velocities and velocity dispersions.

\section{The Ogorodnikov-Milne Model}

The kinematic parameters of the selected stars were determined within the framework of a linear Ogorodnikov-Milne model described in detail by Bobylev et al. (2009). The solar velocity components $U_{\odot}, V_{\odot}, W_{\odot}$ relative to the centroid of the stars under consideration and nine partial derivatives of the velocity with respect to the distance forming the displacement matrix $M$ were determined from the known (trigonometric or photometric) distances $R$, celestial coordinates, radial velocities, and proper motions of the selected stars. In this case, the conditional equations solved by the least-squares method are

$$
\begin{gathered}
V_{r}=-U_{\odot} \cos b \cos l-V_{\odot} \cos b \sin l \\
-W_{\odot} \sin b+R\left(\cos ^{2} b \cos ^{2} l M_{u x}\right. \\
+\cos ^{2} b \cos l \sin l M_{u y}+\cos b \sin b \cos l M_{u z} \\
+\cos ^{2} b \sin l \cos l M_{v x}+\cos ^{2} b \sin ^{2} l M_{v y} \\
+\cos b \sin b \sin l M_{v z}+\sin b \cos b \cos l M_{w x} \\
\left.+\cos b \sin b \sin l M_{w y}+\sin ^{2} b M_{w z}\right) \\
4.74 R \mu_{l} \cos b=U_{\odot} \sin l-V_{\odot} \cos l+ \\
+R\left(-\cos b \cos l \sin l M_{u x}-\cos b \sin ^{2} l M_{u y}-\right. \\
-\sin b \sin l M_{u z}+\cos b \cos ^{2} l M_{v x}+ \\
\left.+\cos b \sin l \cos l M_{v y}+\sin b \cos l M_{v z}\right) \\
4.74 R \mu_{b}=U_{\odot} \cos l \sin b+V_{\odot} \sin \sin b \\
-W_{\odot} \cos b+R\left(-\sin b \cos b \cos { }^{2} l M_{u x}-\right. \\
-\sin b \cos b \sin l \cos l M_{u y}-\sin ^{2} b \cos l M_{u z} \\
-\sin b \cos b \sin l \cos l M_{v x}-\sin b \cos b \sin ^{2} l M_{v y} \\
-\sin b \sin l M_{v z}+\cos { }^{2} b \cos l M_{w x}
\end{gathered}
$$




$$
\left.+\cos ^{2} b \sin l M_{w y}+\sin b \cos b M_{w z}\right) .
$$

We then calculate the Oort constants $A=0.5\left(M_{u y}+M_{v x}\right), B=0.5\left(M_{v x}-\right.$ $\left.M_{u y}\right), C=0.5\left(M_{u x}-M_{v y}\right), K=0.5\left(M_{u x}+M_{v y}\right)$, the vertex deviation $l_{x y}$ : $\tan 2 l_{x y}=-C / A$ and the angular velocity of Galactic rotation $\Omega_{R_{0}}=B-A=$ $-M_{u y}$.

The results of the solution, along with the number of stars, the mean distances $\bar{R}$, and the velocity dispersions $\sigma(U), \sigma(V), \sigma(W)$, are presented in the table for types $\mathrm{K}, \mathrm{M}$, and $\mathrm{C}$ in two variants: with the trigonometric, $R_{\text {hip }}$, and photometric, $R_{p h}$, distances.

We see that the results for $R_{h i p}$ and $R_{p h}$ agree, within the error limits. The Oort constant $A$ and $B$ agree, within the error limits, with the universally accepted ones, for example, those found by Bobylev et al. (2009) for the RGC, $A=15.9 \pm 0.2, B=-12.0 \pm 0.2$. The vertex deviation and the constant $C$ slightly differ from $l_{x y}=7.0 \pm 0.3, C=-3.9 \pm 0.2$ found by Bobylev et al. The constant $K$ shows compression for the set of $K$ stars and expansion for type M. When an admixture of extremely young stars involved in the expansion of the Gould Belt is present among the M stars, this result is explainable and agrees with the result by Bobylev (2004) and the review by Perryman (2009, pp. 324-328).

Comparison of the velocity dispersions and $V_{\odot}$ with the BMG leads us to conclude that the $\mathrm{K}$ and $\mathrm{M}$ stars have similar kinematics typical of disk stars with ages of more than 3 Gyr.

The kinematics of the $\mathrm{C}$ stars is completely different and is typical of disk stars with ages of less than 2 Gyr. Taking into account the spatial distribution of these stars noted above, which may be associated with the Gould Belt, and their positions relative to the isochrones on the $\left(V_{T}-K s\right)$ - $M_{V T}$ diagram (Fig. 2), we can conclude that these are relatively massive $\left(>2 M_{\odot}\right)$ asymptotic branch giants.

Perryman (2009, pp. 449-452) gave an overview of the present ideas about the $\mathrm{C}$ stars including two main groups with a distinctly different evolutionary status. It is hypothesized that the hotter R-subtype carbon stars belong to comparatively old clump red giants with an approximately solar mass, while the cooler N-subtype carbon stars belong to younger asymptotic branch giants with masses of $1.5-4 M_{\odot}$. Knapp et al. (2001) showed that many of the $\mathrm{N}$ stars had so far been erroneously classified as $\mathrm{R}$, although these subtypes must occupy different regions of the color-absolute magnitude diagram. The characteristics of the set of $\mathrm{C}$ stars that we obtained here 
(the uniqueness of the stellar positions on two-color diagrams, the accuracy of the velocities, and the completeness of the sample of 49 selected stars, 41 with velocities, and 46 with spectral classification) confirm that the $\mathrm{C}$ type in the range of colors under consideration actually includes only comparatively young and massive asymptotic branch giants that apparently belong to subtype $\mathrm{N}$ without exception and the applied method is efficient for their identification.

Table 1: Kinematic parameters of the selected stars

\begin{tabular}{l|r|r|r|r|r|r}
\hline & \multicolumn{3}{|c|}{$R_{h i p}$} & \multicolumn{3}{|c}{$R_{p h}$} \\
\hline Parameter & $\mathrm{K}$ & $\mathrm{M}$ & $\mathrm{C}$ & $\mathrm{K}$ & $\mathrm{M}$ & $\mathrm{C}$ \\
\hline Number of stars & 2360 & 685 & 29 & 2737 & 852 & 41 \\
$\bar{R}, \mathrm{pc}$ & 326 & 329 & 459 & 261 & 298 & 273 \\
$\sigma(U), \mathrm{km} \mathrm{s}^{-1}$ & $37 \pm 0.6$ & $40 \pm 0.9$ & $28 \pm 4$ & $34 \pm 0.5$ & $37 \pm 0.7$ & $21 \pm 3$ \\
$\sigma(V), \mathrm{km} \mathrm{s}^{-1}$ & $25 \pm 0.6$ & $30 \pm 0.9$ & $18 \pm 4$ & $27 \pm 0.5$ & $30 \pm 0.7$ & $14 \pm 3$ \\
$\sigma(W), \mathrm{km} \mathrm{s}^{-1}$ & $20 \pm 0.6$ & $22 \pm 0.9$ & $10 \pm 4$ & $20 \pm 0.5$ & $21 \pm 0.7$ & $6 \pm 3$ \\
$\mathrm{U}_{\odot}, \mathrm{km} \mathrm{s}^{-1}$ & $6 \pm 0.6$ & $9 \pm 0.9$ & $13 \pm 4$ & $6 \pm 0.5$ & $9 \pm 0.7$ & $12 \pm 3$ \\
$\mathrm{~V}_{\odot}, \mathrm{km} \mathrm{s}^{-1}$ & $20 \pm 0.6$ & $24 \pm 0.9$ & $8 \pm 4$ & $19 \pm 0.5$ & $23 \pm 0.7$ & $8 \pm 3$ \\
$\mathrm{~W}_{\odot}, \mathrm{km} \mathrm{s}^{-1}$ & $7 \pm 0.6$ & $8 \pm 0.9$ & $6 \pm 4$ & $6 \pm 0.5$ & $7 \pm 0.7$ & $4 \pm 3$ \\
$\mathrm{M}_{u x}, \mathrm{~km} \mathrm{~s}^{-1} \mathrm{kpc}^{-1}$ & $-3 \pm 4$ & $-1 \pm 6$ & $-4 \pm 30$ & $-4 \pm 4$ & $5 \pm 6$ & $32 \pm 20$ \\
$\mathrm{M}_{u y}, \mathrm{~km} \mathrm{~s}^{-1} \mathrm{kpc}^{-1}$ & $29 \pm 4$ & $27 \pm 6$ & $19 \pm 30$ & $33 \pm 4$ & $23 \pm 6$ & $30 \pm 20$ \\
$\mathrm{M}_{u z}, \mathrm{~km} \mathrm{~s}^{-1} \mathrm{kpc}^{-1}$ & $-8 \pm 4$ & $10 \pm 6$ & $15 \pm 30$ & $-14 \pm 4$ & $6 \pm 6$ & $31 \pm 20$ \\
$\mathrm{M}_{v x}, \mathrm{~km} \mathrm{~s}^{-1} \mathrm{kpc}^{-1}$ & $3 \pm 4$ & $1 \pm 6$ & $-10 \pm 30$ & $0 \pm 4$ & $-2 \pm 6$ & $12 \pm 20$ \\
$\mathrm{M}_{v y}, \mathrm{~km} \mathrm{~s}^{-1} \mathrm{kpc}^{-1}$ & $1 \pm 4$ & $10 \pm 6$ & $-11 \pm 30$ & $-8 \pm 4$ & $4 \pm 6$ & $-11 \pm 20$ \\
$\mathrm{M}_{v z}, \mathrm{~km} \mathrm{~s}^{-1} \mathrm{kpc}^{-1}$ & $-5 \pm 4$ & $-5 \pm 6$ & $19 \pm 30$ & $-7 \pm 4$ & $-8 \pm 6$ & $19 \pm 20$ \\
$\mathrm{M}_{w x}, \mathrm{~km} \mathrm{~s}^{-1} \mathrm{kpc}^{-1}$ & $1 \pm 4$ & $-1 \pm 6$ & $-2 \pm 30$ & $2 \pm 4$ & $7 \pm 6$ & $-2 \pm 20$ \\
$\mathrm{M}_{w y}, \mathrm{~km} \mathrm{~s}^{-1} \mathrm{kpc}^{-1}$ & $-1 \pm 4$ & $-2 \pm 6$ & $-8 \pm 30$ & $-5 \pm 4$ & $0 \pm 6$ & $0 \pm 20$ \\
$\mathrm{M}_{w z}, \mathrm{~km} \mathrm{~s}^{-1} \mathrm{kpc}^{-1}$ & $1 \pm 4$ & $2 \pm 6$ & $24 \pm 30$ & $-3 \pm 4$ & $7 \pm 6$ & $17 \pm 20$ \\
$\mathrm{~A}, \mathrm{~km} \mathrm{~s}^{-1} \mathrm{kpc}^{-1}$ & $16 \pm 2$ & $14 \pm 4$ & $4.5 \pm 20$ & $16.5 \pm 2$ & $10.5 \pm 4$ & $21 \pm 15$ \\
$\mathrm{~B}, \mathrm{~km} \mathrm{~s}^{-1} \mathrm{kpc}^{-1}$ & $-13 \pm 2$ & $-13 \pm 4$ & $-14.5 \pm 20$ & $-16.5 \pm 2$ & $-12.5 \pm 4$ & $-9 \pm 15$ \\
$\mathrm{C}, \mathrm{km} \mathrm{s}^{-1} \mathrm{kpc}^{-1}$ & $-2 \pm 2$ & $-5.5 \pm 4$ & $3.5 \pm 20$ & $2 \pm 2$ & $0.5 \pm 4$ & $21.5 \pm 15$ \\
$\mathrm{~K}, \mathrm{~km} \mathrm{~s}^{-1} \mathrm{kpc}^{-1}$ & $-1 \pm 2$ & $4.5 \pm 4$ & $-7.5 \pm 20$ & $-6 \pm 2$ & $4.5 \pm 4$ & $10.5 \pm 15$ \\
$l_{x y}^{\circ}$ & $4 \pm 4$ & $11 \pm 5$ & $-19 \pm 25$ & $-3.5 \pm 2$ & $-1 \pm 5$ & $-23 \pm 15$ \\
$\Omega_{R 0}, \mathrm{~km} \mathrm{~s}^{-1}$ & $-29 \pm 5$ & $-27 \pm 5$ & $-19 \pm 36$ & $-33 \pm 4$ & $-23 \pm 6$ & $-30 \pm 28$ \\
\hline
\end{tabular}




\section{CONCLUSIONS}

Our study showed that the broadband multicolor photometry from the 2MASS and Tycho-2 catalogues is enough to produce a pure (with an admixture of less than 1\%) sample of RGB stars complete within 500 pc of the Sun, to classify the sample stars into types K, M, and C, suspected supergiants, and $\mathrm{S}$ stars, to calculate the interstellar extinction, and to determine the photometric distances of K, M, and C stars with an accuracy of $40 \%$. We showed that our classification is consistent with the old spectral one and, in some cases, is even more accurate.

Tycho-2 proper motions and PCRV radial velocities were used to analyze the stellar kinematics. The decrease in the stellar density distribution with distance from the Galactic equator approximated by the barometric law, contrary to the BMG, and the kinematic parameters calculated using the Ogorodnikov-Milne model characterize the overwhelming majority of the selected $\mathrm{K}$ and $\mathrm{M}$ stars as disk stars with ages of more than 3 Gyr. A small number of $\mathrm{K}$ and $\mathrm{M}$ stars are extremely young or, conversely, thickdisk giants. The latter show a nonuniform distribution in the phase space of coordinates and velocities, arguing against isothermality and full relaxation of the disk and for the theory of dynamical streams or superclusters. The spatial distribution and kinematics of the selected $\mathrm{C}$ stars force us to consider them as young (with ages $<2$ Gyr), fairly massive $\left(>2 M_{\odot}\right)$ asymptotic branch giants probably associated with the Gould Belt. The offset of the Sun above the Galactic equator was found from the distribution of stars to be $13 \pm 2$ pc, which coincides with the previously obtained value for the RGC stars.

\section{ACKNOWLEDGMENTS}

In this study, we used results from the Hipparcos and 2MASS (Two Micron All Sky Survey) projects, the SIMBAD database (http://simbad.ustrasbg.fr/simbad/), and other resources of the Strasbourg Data Center (France), http://cds.u-strasbg.fr/. The study was supported by the "Origin and Evolution of Stars and Galaxies" Program of the Presidium of the Russian Academy of Sciences. 


\section{References}

1. V. Bobylev, Astron. Soc. Pacif. Conf. 316, 224 (2004).

2. V. V. Bobylev, A. S. Stepanishchev, A. T. Bajkova, and G. A. Gontcharov, Pisma Astron. Zh. 35, 920 (2009) [Astronomy Letters 35, 836 (2009)].

3. ESA, Hipparcos and Tycho catalogues (ESA, 1997).

4. B. Famaey, A. Jorissen, X. Luri, et al., Astron. Astrophys. 430, 165, (2005).

5. E.L. Fitzpatrick, D. Massa, Astrophys. J. 663, 320, (2007).

6. L. Girardi, A. Bressan, G. Bertelli, et al., Astron. Astrophys. Suppl. Ser. 141, 371, (2000), http://stev.oapd.inaf.it/YZVAR/.

7. L. Girardi, M.A.T. Groenewegen, E. Hatziminaoglou, et al., Astron. Astrophys. 436, 895, (2005).

8. G. A. Gontcharov, Pisma Astron. Zh. 32, 844 (2006) [Astron. Lett. 32, 759 (2006)]; http://cdsarc.ustrasbg.fr/viz-bin/CatIII/252.

9. G. A. Gontcharov, Pisma Astron. Zh. 34, 10 (2008a) [Astron. Lett. 34, 7 (2008)].

10. G. A. Gontcharov, Pisma Astron. Zh. 34, 868 (2008b) [Astron. Lett. 34, $785(2008)]$.

11. G. A. Gontcharov, Pisma Astron. Zh. 35, 707 (2009a) [Astron. Lett. 35, 638 (2009)].

12. G. A. Gontcharov, Pisma Astron. Zh. 35, 862 (2009b) [Astron. Lett. 35, $780(2009)]$.

13. G. Gontcharov, in Proceedings of the B.V. Kukarkin Centenary Conference on Variable Stars, the Galactic Halo, and Galaxy Formation, Ed. by C. Sterken, N. Samus, and L. Szabados (Mosc. State Univ., Moscow, 2010), p. 161.

14. E. Høg, C. Fabricius, V.V. Makarov, et al., Astron. Astrophys. 355, L27, (2000). 
15. G.R. Knapp, D. Pourbaix, A. Jorissen, Astron. Astrophys. 371, 222, (2001).

16. IRAS working group, IRAS Catalog of Point Sources, Version 2.0 (IRAS, 1988), http://cdsarc.u-strasbg.fr/viz-bin/Cat?II/125.

17. F. van Leeuwen, Astron. Astrophys. 474, 653, (2007).

18. P. P. Parenago, A Course of Stellar Astronomy (GITTL,Moscow, 1954) [in Russian].

19. M. Perryman, Astronomical application of astrometry (Cambridge Univ., Cambridge, 2009).

20. A.J. Pickles, Publ. Astron. Soc. Pacific 110, 863, (1998).

21. A.C. Robin, C. Reyle, S. Derriere, et al., Astron. Astrophys. 409, 523, (2003).

22. M.F. Skrutskie, R.M. Cutri, R. Stiening, et al., Astron. J. 131, 1163, (2006), http://www.ipac.caltech.edu/2mass/releases/allsky/index.html.

23. C. Turon, M. Crézé, D. Egret, et al., Bull. Inform. CDS 43, 5, (1993).

24. B.A. Wichman, I.D. Hill, Applied Statistics 31, 188, (1982).

25. C.O. Wright, M.P. Egan, K.E. Kraemer, et al., Astron. J. 125, 359, (2003). 


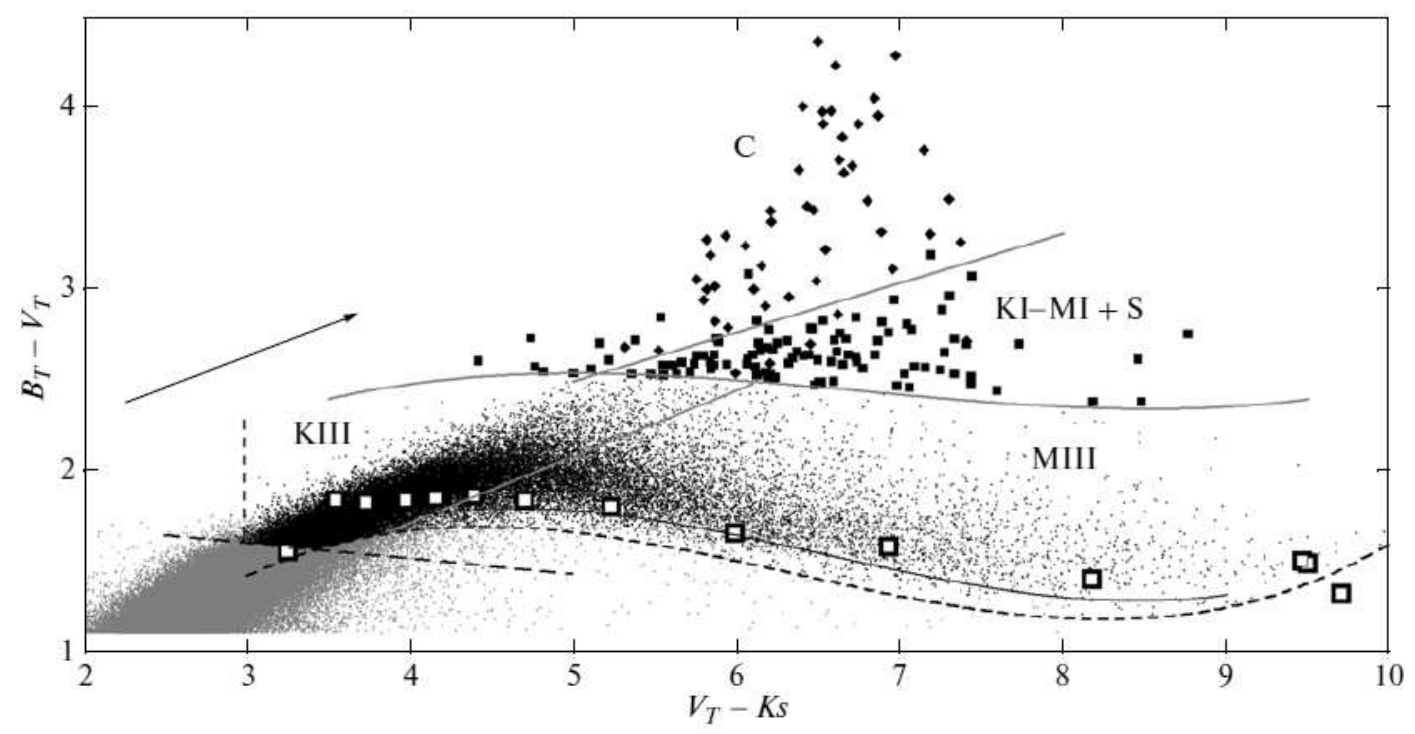

Figure 1: $V_{T}-K s-B_{T}-V_{T}$ diagram: the dashed lines separate the selected RGB stars (black symbols) from the extraneous ones (gray symbols), the large open squares mark the theoretical mean positions of solar-metallicity K3III-M10III stars, the black solid curve indicates the line of normal colors for MIII stars, and the gray lines divide the selected stars into four categories: KIII, MIII, S/supergiants, and type C. 


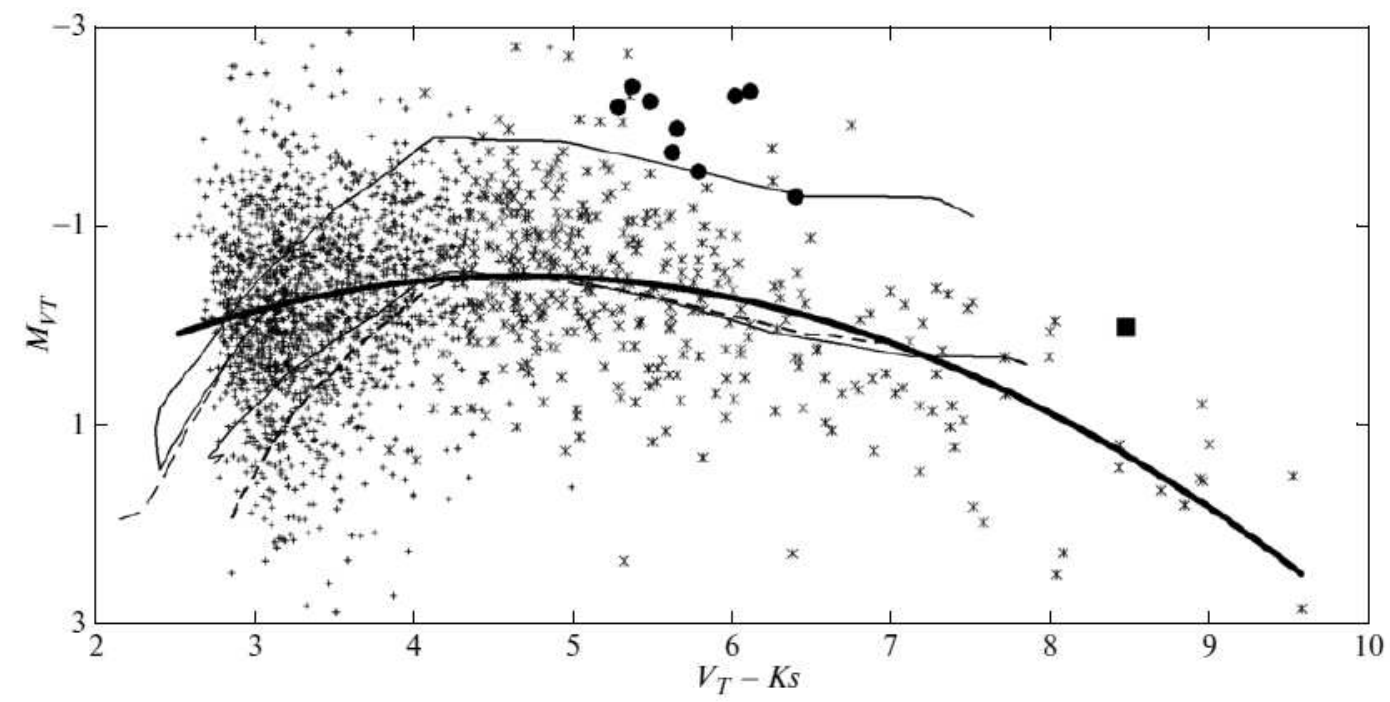

Figure 2: $M_{V T}$ versus $V_{T}-K s$ for the selected $\mathrm{K}$ (crosses), M (snowflakes), $\mathrm{C}$ (circles), and S (square) stars. The thick curve indicates the adopted calibration. The isochrones for solar-metallicity stars are indicated by the thin solid (asymptotic giant branch) and dashed (RGB) curves; the upper solid and dashed lines for an age of $10^{9} \mathrm{yr}$ and the lower one for $10^{10} \mathrm{yr}$. 
(a)
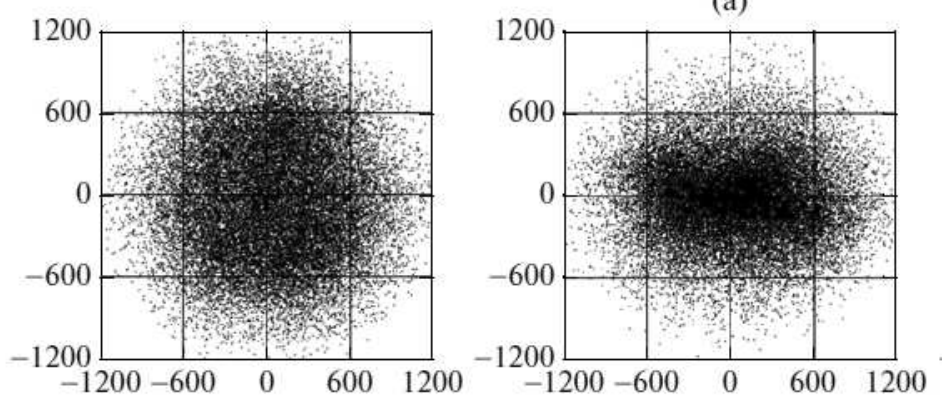

(b)
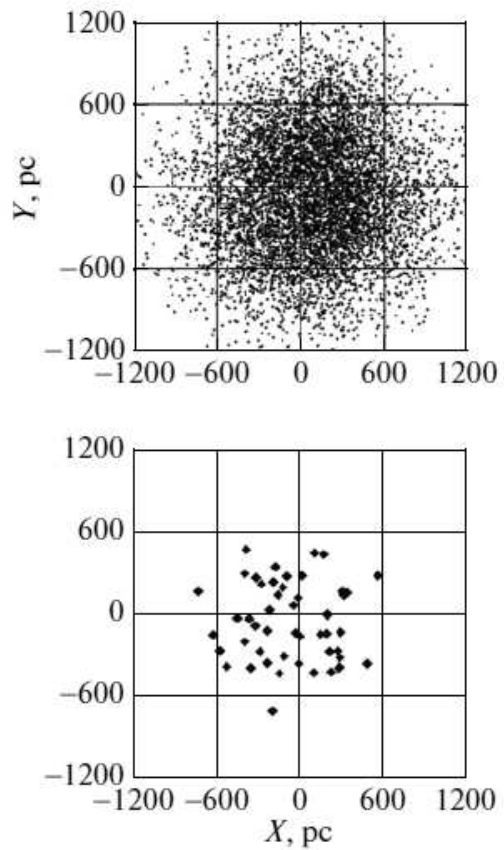

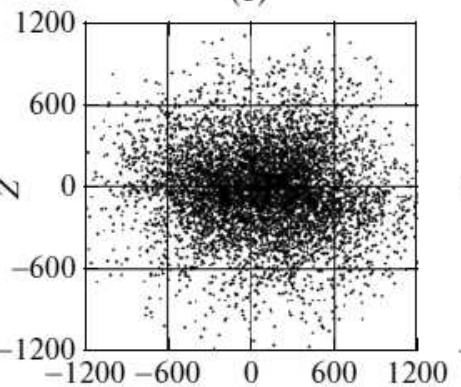

(c)

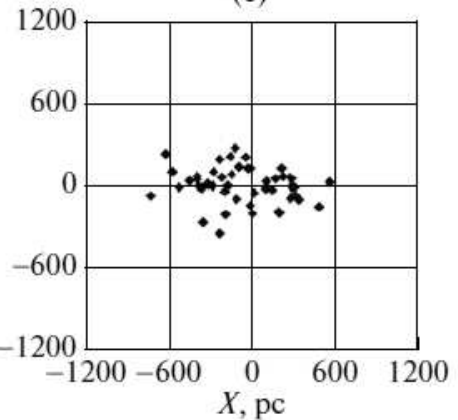

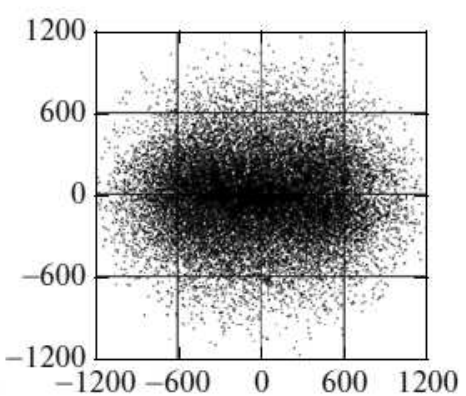
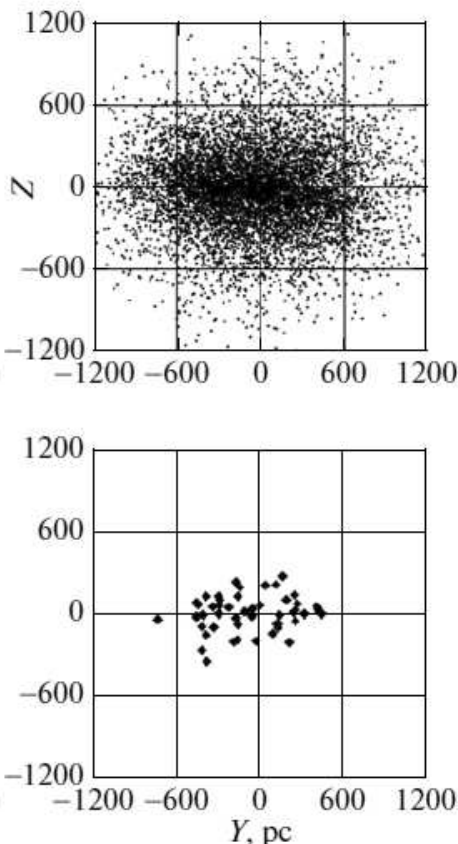

Figure 3: Distribution of the selected stars in projection onto the $X Y, X Z$, and $Y Z$ planes: (a) type $\mathrm{K}$, (b) type $\mathrm{M}$, and (c) type $\mathrm{C}$. 


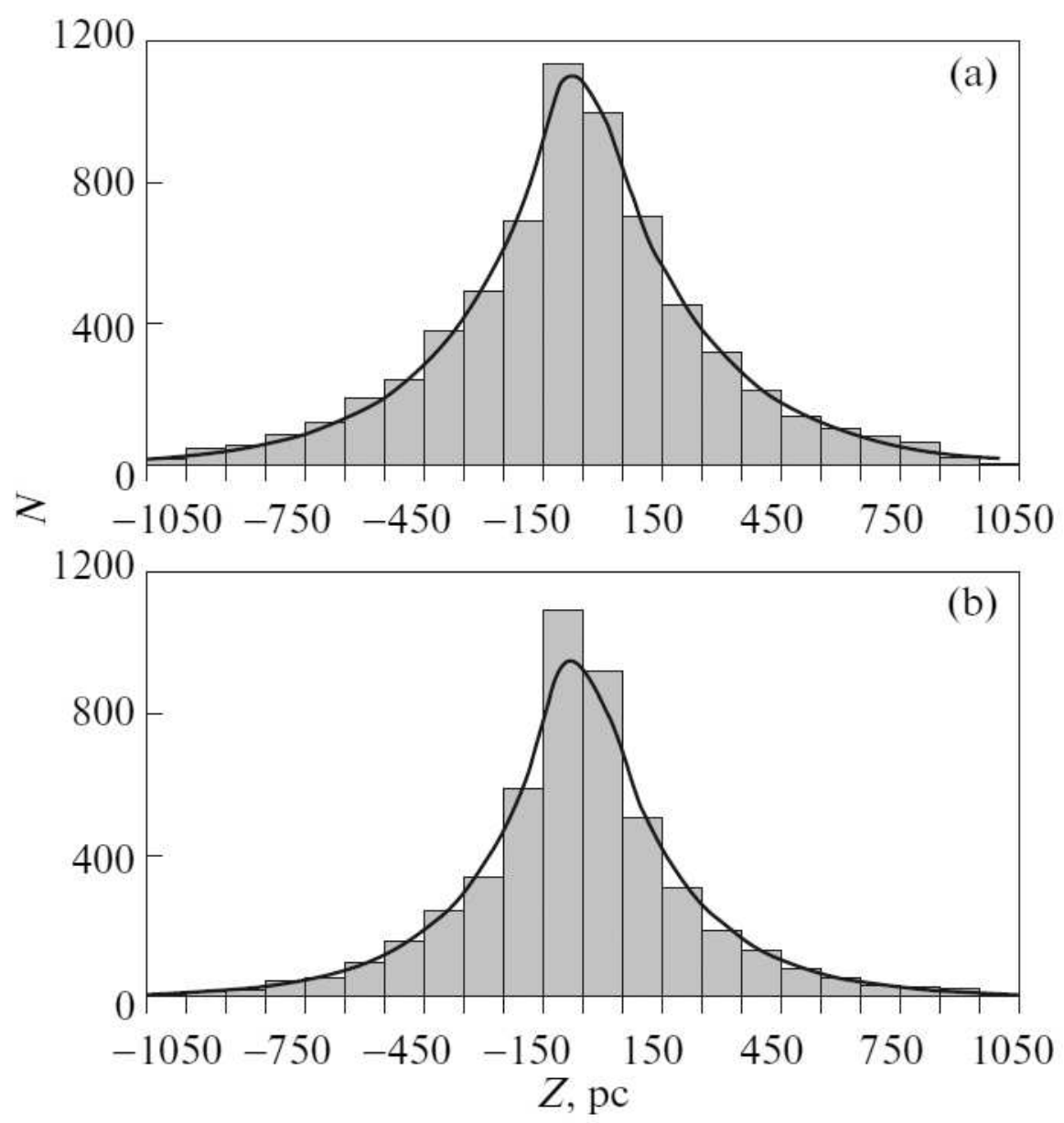

Figure 4: Distribution of the selected (a) $\mathrm{K}$ and (b) $\mathrm{M}$ stars along the $Z$ axis in the vertical cylinder within $400 \mathrm{pc}$ of the Sun; the curves indicate the fit by the barometric law. 

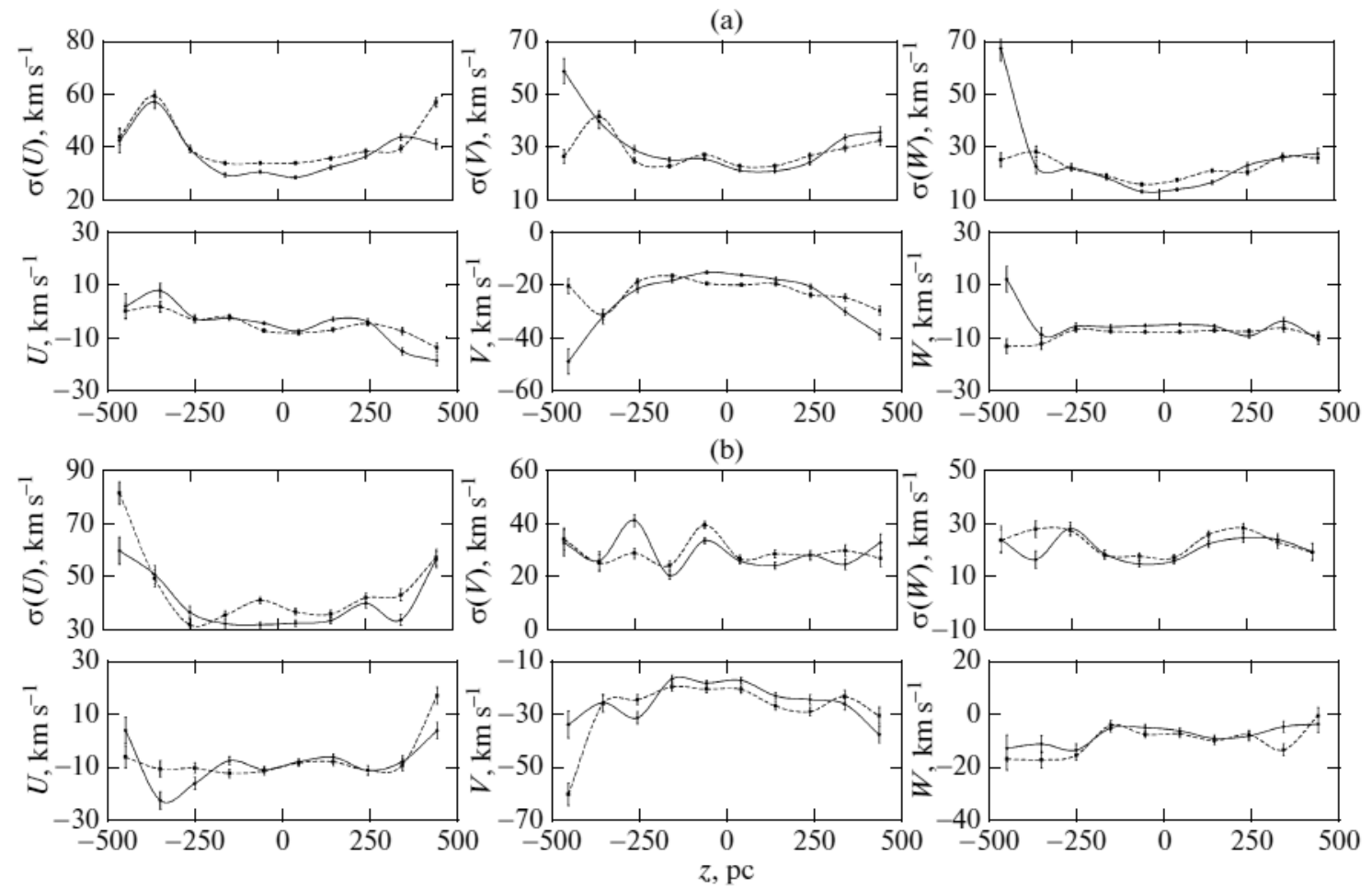

Figure 5: Mean velocity and velocity dispersion in $\mathrm{km} \mathrm{s}^{-1}$ versus $Z$ coordinate in pc in the vertical cylinder elongated along the $Z$ axis within $500 \mathrm{pc}$ of the Sun: (a) type K, (b) type M. The results from photometric distances and Hipparcos parallaxes are indicated by the solid and dashed lines, respectively. 